\title{
Mass Modelling of dwarf Spheroidal Galaxies
}

\author{
Jarosław Klimentowski, ${ }^{1}$ Ewa L. Eokas, ${ }^{1}$ Stelios Kazantzidis, ${ }^{2}$ \\ Francisco Prada, ${ }^{3}$ Lucio Mayer, ${ }^{4,5}$ and Gary A. Mamon ${ }^{6,7}$ \\ ${ }^{1}$ Nicolaus Copernicus Astronomical Center, Bartycka 18, 00-716 Warsaw, Poland \\ ${ }^{2}$ Kavli Institute for Particle Astrophysics and Cosmology, Department of Physics, Stanford \\ University, P.O. Box 20450, M/S 29, Stanford, CA 94309, USA \\ ${ }^{3}$ Instituto de Astrofísica de Andalucia, Apartado Correos 3005, E-18080 Granada, Spain \\ ${ }^{4}$ Institute for Theoretical Physics, University of Zürich, CH-8057 Zürich, Switzerland \\ ${ }^{5}$ Institute of Astronomy, Department of Physics, ETH Zürich, Wolfgang-Pauli Strasse, \\ CH-8093 Zürich, Switzerland \\ ${ }^{6}$ Institut d'Astrophysique de Paris (UMR 7095: CNRS and Université Pierre \& Marie Curie), \\ 98 bis Bd Arago, F-75014 Paris, France \\ ${ }^{7}$ GEPI (UMR 8111: CNRS and Université Denis Diderot), Observatoire de Paris, F-92195 \\ Meudon, France
}

\begin{abstract}
We study the origin and properties of unbound stars in the kinematic samples of dwarf spheroidal galaxies. For this purpose we have run a high resolution $N$-body simulation of a two-component dwarf galaxy orbiting in a Milky Way potential. We create mock kinematic data sets by observing the dwarf in different directions. When the dwarf is observed along the tidal tails the kinematic samples are strongly contaminated by unbound stars from the tails. However, most of the unbound stars can be removed by the method of interloper rejection proposed by den Hartog \& Katgert. We model the velocity dispersion profiles of the cleaned-up kinematic samples using solutions of the Jeans equation. We show that even for such a strongly stripped dwarf the Jeans analysis, when applied to cleaned samples, allows us to reproduce the mass and mass-to-light ratio of the dwarf with accuracy typically better than $25 \%$.
\end{abstract}

Keywords. galaxies: dwarf, galaxies: Local Group, galaxies: kinematics and dynamics, galaxies: fundamental parameters, cosmology: dark matter

\section{Introduction}

Dwarf spheroidal (dSph) galaxies of the Local Group provide critical tests of theories of structure formation in the Universe. The number density of dwarfs in the vicinity of the Milky Way (MW) poses a problem for theories based on Cold Dark Matter (Klypin et al. 1999; Moore et al. 1999). In particular, $\Lambda$ CDM $N$-body simulations predict a few hundred dwarf galaxies in the Local Group while only a few tens are observed. Accurate dwarf mass determination could help in solving this problem.

Galactic dSph galaxies are dark matter (DM) dominated objects. Flat velocity dispersion profiles observed in most of them suggest that they possess extended DM haloes with very high, and increasing with radius, mass-to-light ratios. On the other hand, dSph formation theories based on numerical $N$-body simulations suggest that these objects are products of strong tidal interactions with their host galaxy (Mayer et al. 2001). As a result the extended DM component should be stripped during evolution and we could expect more constant mass-to-light ratios and velocity dispersion profiles not as flat as expected in an extended dark matter halo.

It is still not clear whether observed velocity dispersion profiles are due to the bound population of stars or altered by contamination from stars unbound to the dwarf galaxy. This contamination could be caused by stars stripped from the dSph by tidal interaction 


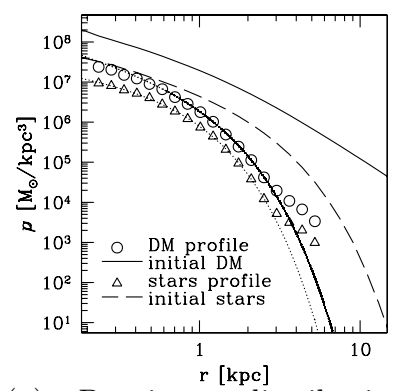

(a) Density distributions of bound stellar and DM particles for the initial conditions and the final stage. Fits to the data points are shown with dotted lines.

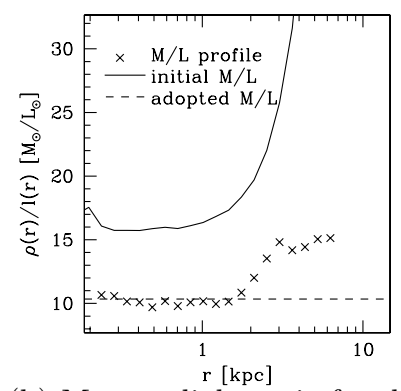

(b) Mass-to-light ratio for the initial conditions and the final stage of the dwarf. The adopted constant $M / L$ ratio is shown with a dashed line.

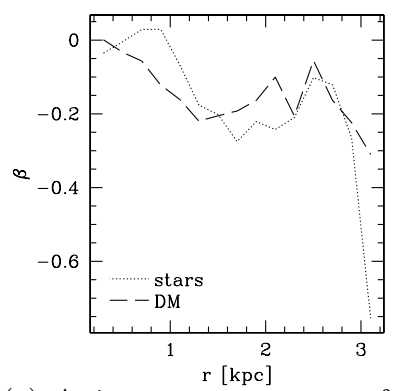

(c) Anisotropy parameter for the stellar and DM particles.

Figure 1. Properties of the simulated dwarf galaxy.

with the host galaxy or by foreground MW stars. There is evidence that unbound stars could be visible in kinematic samples. For example, Coleman et al. (2005) find excess number density of stars around Fornax dwarf depending on the direction of measurement which suggests the presence of tidal tails around this galaxy.

The aim of this work was to study the origin of unbound stars and test the methods of identifying them using $N$-body simulations.

\section{The simulated dwarf}

We ran a high resolution $N$-body simulation based on the 'tidal stirring' scenario of Mayer et al. (2001). A dwarf disk-like stellar system embedded in an NFW (Navarro, Frenk \& White 1997) DM halo was placed on an elongated orbit in a static potential of a MW-sized galaxy. In this approach we neglect the effect of dynamical friction and the response of the primary to the presence of the satellite. However, this choice is justified because orbital decay times are expected to be longer than the Hubble time given the difference in mass between the two systems and the additional mass loss due to tidal stripping (Mayer et al. 2001; Kazantzidis et al. 2004). For more details on the simulation see Klimentowski et al. (2007).

After 10 Gyrs of evolution (corresponding to 5 orbital times) the dwarf loses $99 \%$ of its initial mass and the stellar component forms a spheroid. Figure 1(a) shows the density profiles for both the initial conditions (solid line for DM, dashed for stars) and the final stage (circles for DM, triangles for stars). The data points for the final state were fitted with deprojected Sérsic (1968) profile for stars and the formula proposed by Kazantzidis et al. (2004) for DM particles (dotted lines).

The change in density clearly shows that majority of the mass of the simulated galaxy was lost due to tidal stirring during its evolution. The density profiles for both stars and DM flatten at $r=2.5 \mathrm{kpc}$ from the centre signifying the presence of tidal tails. The DM distribution is similar to the stellar one; as a result the galaxy has a roughly constant mass-to-light ratio in the inner part as indicated in figure 1(b). Figure 1(c) shows the velocity anisotropy parameter for the stellar and DM particles. The orbits are close to isotropic near the centre and weakly tangential in the outer parts. 


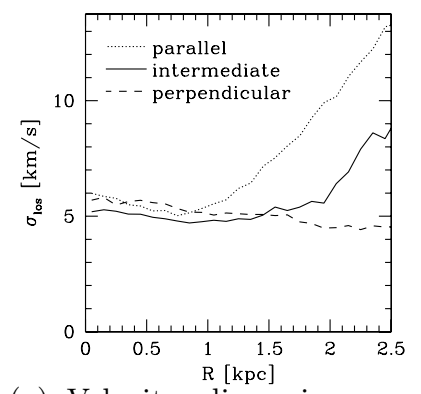

(a) Velocity dispersion profiles for different directions of observation with respect to tidal tails.

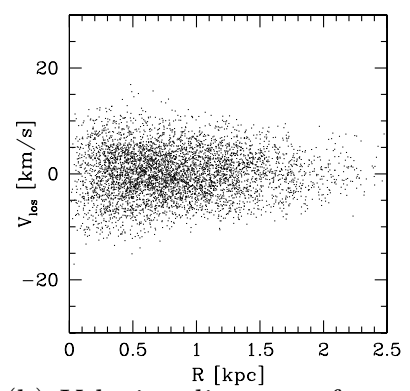

(b) Velocity diagram of stars originating from inside the dwarf $(r<2.5 \mathrm{kpc})$ observed along the tails.

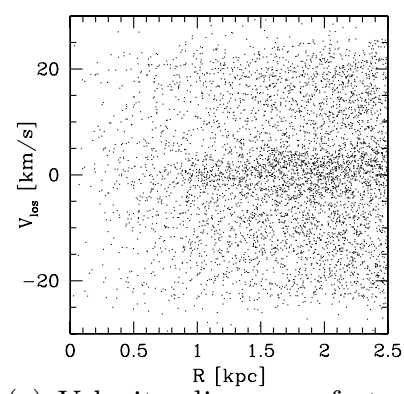

(c) Velocity diagram of stars originating from tidal tails $(r>2.5 \mathrm{kpc})$ observed along the tails.

Figure 2. The origin of contamination by unbound stars and its effect on the velocity dispersion profile.

\section{The contamination by unbound stars}

\subsection{The origin of unbound stars}

We now proceed to create from the simulated data mock kinematic samples by putting an imaginary observer at different locations with respect to the dwarf and its tidal tails and projecting the velocities along the line of sight and radii on the surface of the sky. We introduce an initial cut-off in velocities of $30 \mathrm{~km} \mathrm{~s}^{-1}$ with respect to the dwarf mean velocity. Depending on the angle of view, we measure different velocity dispersion profiles. Figure 2(a) shows velocity dispersion profiles for different angles of view as a function of projected radius. While in the case where the line of sight is perpendicular to the tidal tails the profile is well behaved (slowly decreasing function of radius), it is strongly increasing at higher radii when the tidal tails are viewed along the line of sight ('parallel' case). In order to reproduce this profile via dynamical modelling one would need to assume a very massive and extended DM halo around the galaxy.

The source of this increase of velocity dispersion is the contamination by stars from tidal tails. Figure 2(b) shows a velocity diagram (line-of-sight velocities versus projected radius) of 5000 stars selected from the inside of the dwarf $(r<2.5 \mathrm{kpc})$. All the stars in this region are bound and form a characteristic shape in the velocity diagram typical for virialized objects. Such a sample would produce a well-behaved velocity dispersion profile independently of the direction of observation. On the other hand, figure 2(c) shows a rather uniform velocity distribution of 5000 stars in the tails $(r>2.5 \mathrm{kpc})$ when viewed along the tails. In real observations we cannot separate the two samples and any kinematic sample will contain a mixture of the two with strongest contamination when the line of sight is along the tidal tails. Three examples of such realistic data sets (with 200 stars) are shown in figure 3. Before any attempt at dynamical modelling we need to deal with the unbound stars in a different way.

\subsection{Removal of unbound stars}

In order to remove unbound stars (interlopers) we used the method proposed by den Hartog \& Katgert (1996). The method assumes that a tracer particle (in our case a star in the dwarf galaxy) can be either on a circular orbit with velocity $v_{\text {cir }}=\sqrt{G M(r) / r}$ or falling freely in the galaxy's potential with velocity $v_{\text {inf }}=\sqrt{2} v_{\text {cir }}$ (see den Hartog \& Katgert 1996; Wojtak et al. 2007). We look for the maximum velocity among the two for a given projected radius $R$ and iteratively reject all stars with larger velocities. The mass 


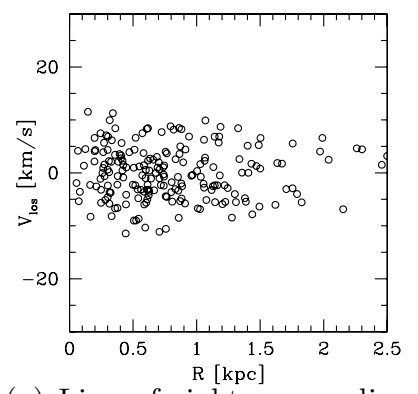

(a) Line of sight perpendicular to the tidal tails.

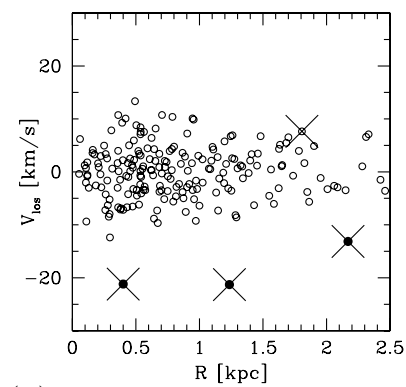

(b) Intermediate direction of view.

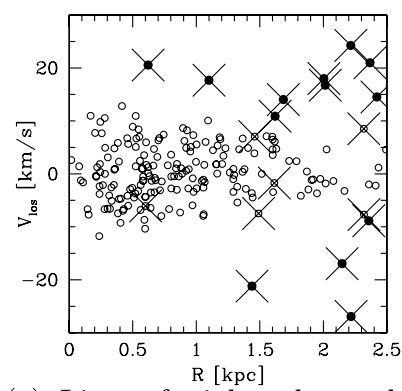

(c) Line of sight along the tidal tails.

Figure 3. Examples of velocity diagrams showing samples of 200 stars selected randomly for the observations performed in different directions: perpendicular to the tidal tails 3(a), along the tails 3(c) and at an intermediate angle 3(b). Unbound stars are marked with crosses. Stars rejected by our procedure of interloper removal are plotted as filled circles, those accepted as open circles.

Table 1. Fractions of removed unbound $\left(f_{i}\right)$ and member (bound) stars $\left(f_{m}\right)$ in percent of the total number of observed stars $(N)$ averaged over 100 velocity diagrams. Numbers are given separately for three directions of observation: along the tidal tails (a), perpendicular to it (c) and an intermediate direction (b).

\begin{tabular}{ccccccc}
\hline$N$ & $f_{i}(\mathrm{a})$ & $f_{m}(\mathrm{a})$ & $f_{i}(\mathrm{~b})$ & $f_{m}(\mathrm{~b})$ & $f_{i}(\mathrm{c})$ & $f_{m}(\mathrm{c})$ \\
\hline 200 & 78.8 & 0.6 & 70.4 & 1.1 & 75.0 & 0.8 \\
400 & 79.4 & 0.7 & 71.4 & 1.2 & 75.8 & 1.1 \\
800 & 80.4 & 0.7 & 71.3 & 1.2 & 77.5 & 1.1 \\
\hline
\end{tabular}

profile needed for the calculation of the velocities is estimated in each iteration using the standard mass estimator $M_{V T}$ derived from the virial theorem (Heisler et al. 1985)

$$
M_{V T}\left(r=R_{\max }\right)=\frac{3 \pi N}{2 G} \frac{\Sigma_{i}\left(V_{i}-\bar{V}\right)^{2}}{\sum_{i<j} 1 / R_{i, j}},
$$

where $N$ is a number of stars with projected radii $R<R_{\max }, V_{i}$ is the line-of-sight velocity of the $i$-th star and $R_{i, j}$ is a projected distance between $i$-th and $j$-th star. The mass profile can be simply obtained as $M(r) \approx M_{V T}\left(R_{i}<r<R_{i+1}\right)$, where $R_{i}$ is the sequence of projected radii of stars in the increasing order.

We repeated the procedure for realistic velocity diagrams with different numbers of stars and angles of view. Overall, the method works very well rejecting on average between 70 and $80 \%$ of unbound stars and very few bound ones. Its performance is illustrated and summarized in the example diagrams of figure 3 and table 1.

\section{Modelling of the velocity dispersion}

Jeans formalism is the main tool in mass modelling of dwarf spheroidal galaxies. Our simulated dSph galaxy has a mass-to-light ratio and velocity anisotropy almost constant with radius so the measured velocity dispersion profile can be reproduced by just two constant parameters $M / L$ and $\beta$. Following Eokas et al. (2005, 2006) we therefore fit these two parameters by adjusting to the data a solution of the Jeans equation

$$
\sigma_{\operatorname{los}}^{2}(R)=\frac{2}{\Sigma(R)} \int_{R}^{\infty} \frac{\nu \sigma_{r}^{2} r}{\sqrt{r^{2}-R^{2}}}\left(1-\beta \frac{R^{2}}{r^{2}}\right) \mathrm{d} r .
$$


Table 2. Parameters fitted to the velocity dispersion profiles, the mass-to-light ratio $M / L$ (corresponding to mass $M$ ) and anisotropy $\beta$, averaged over 100 kinematic samples cleaned of interlopers for observations performed in three directions: along the tidal tails (a), at an intermediate angle (b) and perpendicular to tidal tails (c). The first row lists values obtained from the $3 \mathrm{D}$ simulation data.

\begin{tabular}{cccc}
\hline & $M / L_{\mathrm{fit}}\left[\mathrm{M}_{\odot} / \mathrm{L}_{\odot}\right]$ & $M\left[10^{7} M_{\odot}\right]$ & $\beta_{\mathrm{fit}}$ \\
\hline $3 \mathrm{D}$ & 10.3 & 4.0 & -0.13 \\
$\mathrm{a}$ & $7.56 \pm 0.88$ & $3.5 \pm 0.4$ & $-0.02 \pm 0.30$ \\
$\mathrm{~b}$ & $8.02 \pm 1.17$ & $3.2 \pm 0.5$ & $-0.46 \pm 0.44$ \\
$\mathrm{c}$ & $9.69 \pm 1.00$ & $3.7 \pm 0.4$ & $-0.33 \pm 0.26$ \\
\hline
\end{tabular}

The results of the fitting for 300 kinematic samples of 200 stars each (100 in each direction of observation) are summarized in table 2 .

The results are in best agreement with the true values measured from $3 \mathrm{D}$ data in the case (c). In cases (a) and (b) the mass-to-light ratio is slightly underestimated. However, this underestimation is not due to inaccuracies in the performance of our interloper removal scheme. We verified that the dispersion profiles measured in case (c) agree well with the dispersion profile of bound stars in the dwarf so are fully reliable. Instead, the dispersion measured along the tails turns out to be smaller than in other directions which is probably due to some stars being lost in tidal stripping.

\section{Conclusion}

We used an $N$-body simulation to assess reliability of dynamical mass modelling of dwarf spheroidal galaxies. We found that even strongly tidally stripped objects can be reliably modelled after a careful removal of unbound stars from kinematic samples. We tested a method for dealing with interlopers and found its efficiency to be up to $80 \%$. This procedure combined with modelling of the velocity dispersion profile by standard solutions of the Jeans equation allows us to measure masses of dSph galaxies with typical accuracy better than $25 \%$.

\section{References}

Coleman, M. G., Da Costa, G. S., Bland-Hawthorn, J., \& Freeman, K. C. 2005 AJ, 129, 1443 den Hartog, R., \& Katgert, P. 1996, MNRAS, 279, 349

Heisler, J., Tremaine, S., \& Bahcall, J. N. 1985, ApJ, 298, 8

Kazantzidis, S., Mayer, L., Mastropietro, C., Diemand, J., Stadel, J., \& Moore, B. 2004, ApJ, 608,663

Klimentowski, J., Łokas, E. L., Kazantzidis, S., Prada, F., Mayer, L., \& Mamon, G. A. 2007, MNRAS, 378, 353

Klypin, A., Kravtsov, A. V., Valenzuela, O., \& Prada, F. 1999, ApJ, 522, 82

Łokas, E. L., Mamon, G. A., \& Prada, F. 2005, MNRAS, 363, 918

Łokas, E. L., Mamon, G. A., \& Prada, F. 2006, in: G.A. Mamon et al. (eds.), Mass Profiles and Shapes of Cosmological Structures, EAS Publications Series, Volume 20, p. 113

Mayer, L., Governato, F., Colpi, M., Moore, B., Quinn, T., Wadsley, J., Stadel, J., \& Lake, G. 2001, ApJ, 559, 754

Moore, B., Ghigna, S., Governato, F., Lake, G., Quinn, T., Stadel, J., \& Tozzi, P. 1999, ApJ, L19

Navarro, J. F., Frenk, C. S., \& White, S. D. M. 1997, ApJ, 490, 493

Sérsic, J. L. 1968, Atlas de Galaxies Australes, Observatorio Astronomico, Cordoba

Wojtak, R., Łokas, E. L., Mamon, G. A., Gottlöber, S., Prada, F., \& Moles, M. 2007, A\&A, 466,437 